\title{
Does Risk Aversion Accelerate Optimal Forest Rotation Under Uncertainty?
}

\author{
Luis H. R. Alvarez \\ Turku School of Economics and Business Administration \\ and \\ Erkki Koskela \\ University of Helsinki, RUESG and HECER
}

Discussion Paper No. 21

September 2004

ISSN 1795-0562

HECER - Helsinki Center of Economic Research, P.O. Box 17 (Arkadiankatu 7), Fl-00014 University of Helsinki, FINLAND, Tel +358-9-191-28780, Fax +358-9-191-28781, E-mail info-hecer@helsinki.fi, Internet www.hecer.fi 


\title{
Does Risk Aversion Accelerate Optimal Forest Rotation Under Uncertainty?*
}

\begin{abstract}
We use a Wicksellian single rotation framework to analyze the impact of the intertemporally fluctuating and stochastic mean-reverting interest rate process on the optimal harvesting threshold and thereby the expected length of the rotation period, when forest value is also stochastic following geometric Brownian motion and landowners are risk averse. We provide an explicit solution for the two-dimensional path-dependent rotation problem and demonstrate that higher interest rate volatility increases, while higher risk aversion decreases the optimal harvesting threshold. Moreover, under risk aversion increased forest value volatility decreases the optimal harvesting threshold, while it has no effect under risk neutrality. Numerical illustrations indicate that higher interest rate volatility will raise the expected rotation period at an increasing rate, while higher forest value volatility will decrease its sensitivity under risk aversion.
\end{abstract}

JEL Classification: Q23, G31, C61.

Keywords: Forest rotation, risk aversion, stochastic interest rate, optimal stopping.

Luis H. R. Alvarez

Department of Economics,

Quantitative Methods in Management

Turku School of Economics and

Business Administration

FI-20500 Turku

FINLAND

e-mail: luis.alvarez@tukkk.fi
Erkki Koskela

Department of Economics

P.O. Box 17 (Arkadiankatu 7)

University of Helsinki

FI-00014 University of Helsinki

FINLAND

e-mail: erkki.koskela@helsinki.fi

* The research of Luis H. R. Alvarez has been supported by the Foundation for the Promotion of the Actuarial Profession and the Finnish Insurance Society. Erkki Koskela thanks the Research Unit of Economic Structures and Growth (RUESG) in the University of Helsinki and the Yrjö Jahnsson Foundation for financial support and the Research Department of the Bank of Finland for hospitality. 


\section{Introduction}

The most often used starting point in the analyzes of optimal rotation period of even age forest stands has been the Faustmannian ongoing rotations framework. Under the assumptions of constant exogenous parameters and perfect capital markets the basic deterministic model leads to a constant optimal rotation period for an even age stand to maximize the present value of forest stand (see e.g. Johansson and Löfgren 1985 and Samuelson 1976). The deterministic framework has been extended in studies focusing the impacts of risk of forest fire (see e.g. Reed 1984), stochastic forest stand value (see e.g. Clarke and Reed 1989, 1990, Willassen 1998, Alvarez 2004 and Chang 2004) and stochastic timber prices (see e.g. Insley 2002 and Plantinga 1998) on the optimal harvesting threshold and on the expected rotation length. Modelling forest fire risk as a Poisson process implies a shorter rotation period than in the deterministic case due to the higher effective discount rate for forestry (cf. Reed 1984). But usually the reverse happens in the presence of stochastic forest stand value and timber price; higher volatility will increase the optimal harvesting threshold and thereby lengthen the expected rotation period. The explanation of this observation goes as follows. Even though higher volatility raises the expected net present value of the harvesting yield, it also raises the value of waiting by increasing the expected net present value of the future harvesting opportunities. Since the latter effect dominates the former the net impact of increased volatility on the optimal harvesting threshold is unambiguously positive (see e.g. Clarke and Reed 1989, 1990, Willassen 1998, Alvarez 2004 and Chang 2004).

All studies mentioned above have used the assumption of constant and deterministic discount rate, which is problematic because rotation periods are usually quite long and interest rates fluctuate over time. It is known on the basis of extensive empirical research in financial economics (see e.g. Cochrane 2001, Ch. 20) that in the long run interest rates are volatile and follow mean-reverting processes. Alvarez and Koskela 2003, 2004 have used a Wicksellian single rotation framework to analyze the impact of the intertemporally fluctuating and stochastic mean-reverting interest rate process on the optimal harvesting threshold and the expected length of rotation period, when forest stand value has also been assumed to be stochastic and landowners are risk neutral. In this paper we extend these analyzes in the two dimensions. First, we model the interest rate as a more general parametrized mean-reverting process using the well-known Cox-Ingersoll-Ross 1985 model which provides a very realistic specification of the interest rate process over time. We model forest stand value, i.e. the product of timber price and forest volume, as a geometric Brownian motion. Second, and importantly, we assume that landowners are risk averse and ask: what is the potential role of risk aversion under stochastic interest rate and stochastic forest stand value? 
In this paper we provide the following new results. First, in the case of risk-averse landowners we show an explicit solution for the tree-cutting problem under interest rate and forest value uncertainty by expressing the original path-dependent optimal stopping problem as an associated ordinary path-independent optimal stopping problem. Second, we demonstrate that higher interest rate volatility increases the optimal harvesting threshold of a risk-averse landowner and therefore lengthens the expected rotation period. Third, higher relative risk aversion decreases the optimal harvesting threshold and thus shortens the expected rotation period. Fourth, under risk aversion increased forest value volatility decreases the optimal harvesting threshold, but it has no effect under risk neutrality. Finally, numerical illustrations indicate that higher interest rate volatility raises the optimal harvesting threshold and the expected rotation period at an increasing rate, while higher forest value volatility will decrease the sensitivity of the optimal harvesting threshold with respect to interest rate volatility under risk averse landowners.

We proceed as follows. In section 2 we present and solve a two-dimensional and pathdependent optimal stopping problem in terms of the harvesting threshold under stochastic interest rate and stochastic forest value when landowner behavior is risk averse. Moreover, we illustrate our results numerically. Finally, there is a brief concluding section.

\section{Optimal Forest Rotation Under Interest Rate and Forest Stand Value Uncertainty}

In this section we establish the following results. First, we characterize the optimal rotation problem under stochastic interest rate and forest stand value and show that under a set of plausible assumptions the two-dimensional path-dependent rotation problem can be re-expressed as an ordinary path-independent optimal stopping problem. Second, we demonstrate that the transformed rotation problem is explicitly solvable and provide an analytic characterization. Third, we show that higher interest rate volatility decelerates, whil higher relative risk aversion accelerates rotation. Finally, a numerical illustration about the relationship between the optimal rotation threshold and interest rate volatility and its dependence on risk aversion is also presented.

Consider the following (path-dependent) Wicksellian optimal rotation problem

$$
V_{\gamma}(x, r)=\sup _{\tau} \mathbf{E}_{(x, r)}\left[e^{-\int_{0}^{\tau} r_{s} d s} u\left(X_{\tau}\right)\right]
$$

where the underlying timber value and interest rate processes $\left(X_{t}, r_{t}\right)$ evolve according to the 
dynamics described by the following stochastic differential equations

$$
d r_{t}=\left(a-b r_{t}\right) d t+c \sqrt{r_{t}} d W_{t}, \quad r_{0}=r
$$

and

$$
d X_{t}=\mu X_{t} d t+\sigma X_{t} d \hat{W}_{t}, \quad X_{0}=x
$$

where $a, b, c, \sigma, \mu \in \mathbb{R}_{+}$are known exogenously given constants, and $W_{t}$ and $\hat{W}_{t}$ are two stochastically independent Wiener processes (under the objective probability measure $\mathbb{P}$ ). In what follows we assume that the utility function is of the standard HARA-type (cf. Merton 1971) $u(x)=\frac{1}{\gamma} x^{\gamma}$, where $1-\gamma \in(0,1)$ is the coefficient measuring the rate of relative risk aversion. The interest rate $r_{t}$ follows a mean-reverting process while the timber value $X_{t}$ follows a geometric Brownian motion. It is worth emphasizing - as we mentioned earlier that the interest rate model (2.2) is known in financial economics as the Cox-Ingersoll-Ross model of the interest rate which can be supported theoretically (cf. Cox, Ingersoll, and Ross 1985) and which lies in conformity with empirics (cf. Björk 1998, chapter 17, and Cochrane 2001 , chapters 19,20$)$. It is also worth pointing out that if $a \geq c^{2} / 2$, then the interest rate process $r_{t}$ converges towards a long run stationary (Gamma-) distribution with density (cf. Borodin and Salminen 2002, pp. 35-37)

$$
p(r)=(b \eta)^{a \eta} \frac{r^{a \eta-1} e^{-b \eta r}}{\Gamma(a \eta)},
$$

where $\eta=2 / c^{2}>0$. Especially, we find that if $a \geq c^{2} / 2$ then the expected long-run interest rate can be expressed as $\lim _{t \rightarrow \infty} \mathbf{E}\left[r_{t}\right]=a / b>0$ which coincides with the long run stationary steady state interest rate in the absence of uncertainty.

Before proceeding in the analysis of the stochastic valuation and forest rotation, we first establish the following benchmark outcome, characterized in

Theorem 2.1. In the absence of volatility of interest rate and forest stand value, i.e. when $c=\sigma=0$ and assuming that $\mu \gamma<a / b$, which guarantees the finiteness of the value of the optimal policy, the optimal rotation date is

$$
t^{*}=\ln \left(\frac{a-b \min (\mu \gamma, r)}{a-b \mu \gamma}\right)^{1 / b}
$$

and the value of the optimal rotation strategy can be expressed as

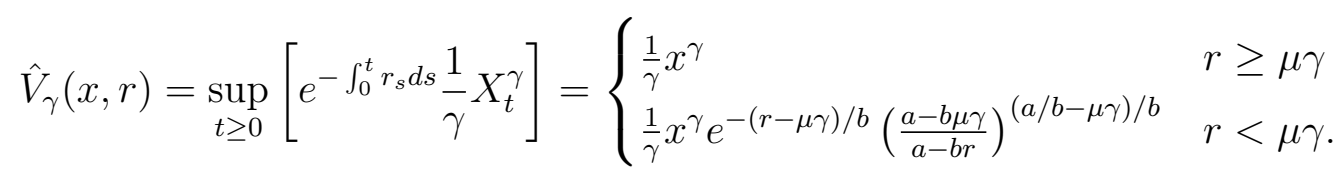


Proof. See Appendix A.

It is worth noticing that the optimal rotation date $t^{*}$ is the first date at which the underlying interest rate process $r_{t}$ hits the growth rate $\mu \gamma$ from below. The absence of speculative bubbles condition guarantees that this date is finite and, therefore, that the forest will eventually be harvested depending on the relative sizes of the parameters. Especially, we find that an increase in the per capita growth rate of the forest stand value prolongs the length of the optimal rotation period $t^{*}$ by increasing the required rate of return. Somewhat interestingly, the optimal rotation policy does not depend on the forest stand value (except for its growth rate) but is solely determined by the underlying interest rate dynamics described by parameters $a$ and $b$. This observation is based on the constancy (as a function of the current forest stand value $x$ ) of the ratio $\hat{V}_{\gamma}(x, r) / x^{\gamma}$. Importantly, we observe that the optimal harvesting threshold $\mu \gamma$ is an increasing function of the parameter $\gamma$ implying that increased relative risk aversion (i.e. a decrease in $\gamma$ ) decreases the optimal harvesting threshold and, therefore, has an accelerating effect on optimal rotation.

Having characterized the underlying stochastic dynamics in (2.2) and (2.3) and the optimal single rotation problem (2.1) in the absence of volatility we can now state the following important result

Lemma 2.2. Under the stochastic interest rate and forest stand value dynamics (2.2) and (2.3) the path-dependent optimal rotation problem (2.1) can be re-expressed as the following ordinary path-independent optimal stopping problem

$$
V_{\gamma}(x, r)=\frac{1}{\gamma} x^{\gamma} e^{A r} \sup _{\tau} \mathbf{E}_{r}\left[e^{(\delta(\gamma)+a A) \tau-A \hat{r}_{\tau}}\right]
$$

where

$$
A=\frac{b}{c^{2}}-\sqrt{\frac{b^{2}}{c^{4}}+\frac{2}{c^{2}}}<0
$$

denotes the negative root of the quadratic equation $c^{2} z^{2}-2 b z-2=0, \delta(\gamma)=\mu \gamma+\frac{1}{2} \sigma^{2} \gamma(\gamma-1)$, and the interest rate $\hat{r}_{t}$ evolves (under $\mathbb{P}$ ) according to the dynamics described by the stochastic differential equation

$$
d \hat{r}_{t}=\left(a-\left(b-A c^{2}\right) \hat{r}_{t}\right) d t+c \sqrt{\hat{r}_{t}} d W_{t}, \quad \hat{r}_{0}=r .
$$

Especially, problem (2.5) is independent of forest stand value volatility $\sigma$ under risk neutrality (i.e. when $\gamma=1$ ).

Proof. See Appendix B. 
Lemma 2.2 is crucial in the sense that using this we can demonstrate that under the assumptions concerning the stochastic processes modelling the interest rate and the forest stand value we get an ordinary and solvable one-dimensional optimal stopping problem. It is worth observing that our finding is essentially based on a technique known as a change of numeraire (cf. Björk, 1998 chapter 19). More precisely, instead of tackling the original valuation directly, we simplify the analysis by expressing the value of the project in terms of the price of a zero coupon bond maturing at exercise. Our main new result is now summarized in the following

Theorem 2.3. Assume that the absence of speculative bubbles condition $\delta(\gamma)+a A<0$, guaranteeing the finiteness of the value of the optimal policy, is satisfied. Then the value of the optimal single rotation problem (2.1) reads as

$$
V_{\gamma}(x, r)=\frac{1}{\gamma} x^{\gamma} e^{A r} \psi(r) \sup _{y \geq r}\left[\frac{e^{-A y}}{\psi(y)}\right]= \begin{cases}\frac{1}{\gamma} x^{\gamma}, & r \geq r^{*} \\ \frac{1}{\gamma} x^{\gamma} e^{A\left(r-r^{*}\right)} \frac{\psi(r)}{\psi\left(r^{*}\right)}, & r<r^{*}\end{cases}
$$

where the increasing fundamental solution

$$
\psi(r)=\int_{0}^{1} e^{2\left(b-A c^{2}\right) r t / c^{2}} t^{\rho-1}(1-t)^{2 a / c^{2}-\rho-1} d t
$$

is known as Kummer's confluent hypergeometric function (see e.g. Abramowitz and Stegun 1968, pp. 503-535) and $\rho=(\delta(\gamma)+a A) /\left(A c^{2}-b\right)>0$. The optimal interest rate exercise threshold $r^{*}$ is the unique root of the ordinary first order condition $\psi^{\prime}\left(r^{*}\right)=-A \psi\left(r^{*}\right)$ and it has the following properties: $r^{*}>\mu \gamma$ for $c>0$ and $r^{*}=\mu \gamma$ when $c=0$. Moreover, under risk neutrality (i.e. when $\gamma=1$ ) the optimal rotation policy is independent of the forest stand value volatility $\sigma$.

Proof. See Appendix C.

Theorem 2.3 demonstrates that the path-dependent optimal rotation problem (2.5) is explicitly solvable whenever the absence of speculative bubbles condition $\delta(\gamma)+a A<0$ - guaranteeing the finiteness of the value of the optimal policy - is satisfied. In order to qualify the combined effect of interest rate and forest stand value volatility on the absence of speculative bubbles condition consider the mapping $f(c, \sigma)=\delta(\gamma)+a A$. Since $\lim _{c \downarrow 0} f(c, \sigma)=$ $\delta(\gamma)-a / b, \lim _{c \rightarrow \infty} f(c, \sigma)=\delta(\gamma)$, and

$$
f_{c}(c, \sigma)=\frac{c a A^{2}}{b-c^{2} A}>0
$$

we immediately observe that if $\delta(\gamma) \geq a / b$ then the absence of speculative bubbles condition $\delta(\gamma)+a A<0$ is never satisfied and in that case the value of the harvesting opportunity 
becomes unbounded. On the other hand, if $\delta(\gamma) \leq 0$ then the absence of speculative bubbles condition $\delta(\gamma)+a A<0$ is satisfied for all values of interest rate volatility. However, if $0<\delta(\gamma)<a / b$, then there is a unique critical volatility coefficient

$$
c^{*}=\sqrt{\frac{2 a b}{\delta^{2}(\gamma)}\left(\frac{a}{b}-\delta(\gamma)\right)}>0
$$

above which the the absence of speculative bubbles condition $\delta(\gamma)+a A<0$ is again violated. Interestingly, we find that

$$
\frac{\partial c^{*}}{\partial \sigma}=\frac{\sigma \gamma(1-\gamma)\left(b-c^{2} A\right)}{c a A^{2}}>0
$$

This means that the critical interest rate volatility coefficient is an increasing function of forest stand value volatility under risk aversion. In other words, the set of admissible interest rate volatilities expands as forest stand value volatility increases.

In order to analyze the effect of forest stand value volatility on the absence of speculative bubbles condition, we first notice that under risk aversion $f(c, 0)=\gamma \mu+a A, \lim _{\sigma \rightarrow \infty} f(c, \sigma)=$ $-\infty$, and $f_{\sigma}(c, \sigma)=\sigma \gamma(\gamma-1)<0$ implying that the absence of speculative bubbles condition is satisfied for all forest stand value volatilities whenever the condition $\gamma \mu+a A \leq 0$ is satisfied. However, if $\gamma \mu+a A>0$, then there is a critical volatility coefficient

$$
\sigma^{*}=\sqrt{\frac{2(a A+\mu \gamma)}{\gamma(1-\gamma)}}>0
$$

below which the absence of speculative bubbles condition is violated. In other words, if the condition $\gamma \mu+a A>0$ is satisfied, then the value of the harvesting opportunity is finite only when forest stand value volatility is sufficiently high.

As is clear from $(2.7)$, the factor $\delta(\gamma)+a A$ is an increasing function of interest rate volatility. Hence, higher interest rate volatility increases the required exercise premium and, thus, prolongs the expected length of the optimal rotation period. An economic interpretation of this finding goes as follows. Higher interest rate volatility increases the certainty-equivalent interest rate and thereby lengthens the optimal rotation period. In financial terms, increased interest rate volatility increases the value of the harvesting opportunity $V_{\gamma}(x, r)$ (by increasing the value of zero-coupon bonds maturing at the exercise date $\tau$ ) while leaving the exercise payoff $\frac{1}{\gamma} x^{\gamma}$ unaffected. However, since the option to harvest is lost at exercise (by the usual balance identity $V_{\gamma}\left(x, r^{*}\right)=\frac{1}{\gamma} x^{\gamma}$ stating that at the optimum the project value should be equal to its full cost which in this case is the lost option value), we observe that increased interest rate volatility raises the required exercise premium and, therefore, prolongs the expected length of the optimal rotation period.

The impact of risk aversion on the optimal rotation policy and its value is now summarized in the following. 
Theorem 2.4. If $\mu \geq \frac{1}{2} \sigma^{2}$ then the optimal rotation threshold is an increasing function of the parameter $\gamma$. That is, if the condition $\mu \geq \frac{1}{2} \sigma^{2}$ is satisfied then increased relative risk aversion shortens the expected length of the rotation period by decreasing the optimal harvesting threshold.

Proof. See Appendix D.

Along the lines of our findings in the deterministic setting, Theorem 2.4 demonstrates that higher relative risk aversion accelerates optimal rotation by decreasing the optimal harvesting threshold. This observation is of interest since it emphasizes the inter-temporal aspect of risk aversion. Put somewhat differently, a rational risk averse agent prefers less uncertain returns realized in the near future in comparison with potentially higher returns realized in the far future and subject to larger uncertainty. A second key comparative static property of the optimal policy and its value is now summarized in the following.

Theorem 2.5. Under risk aversion, increased forest stand value volatility decreases the value of the optimal harvesting policy and accelerates rotation by decreasing the optimal harvesting threshold. Put formally, $\partial V_{\gamma}(x, r) / \partial \sigma<0$ and $\partial r^{*} / \partial \sigma<0$ whenever $0<\gamma<1$.

Proof. See Appendix E.

According to Theorem 2.5 under risk aversion, both the optimal harvesting threshold and the value of the harvesting opportunity are decreasing functions of forest stand value volatility. Thus, in such circumstances increased forest stand value volatility unambiguously accelerates optimal rotation. This result is of interest, since the optimal harvesting policy and its value are independent of forest stand value volatility under risk neutrality. Hence, in the present case the accelerating effect of higher relative risk aversion is strengthened by the presence of forest stand value volatility.

The impact of relative risk aversion on the sensitivity of the optimal harvesting threshold with respect to changes in the volatility of the underlying interest rate dynamics is illustrated in Figure 1 under the assumptions that $b=0.1, a=0.045 b$, and $\mu=0.03$.

The impact of forest stand value volatility on the sensitivity of the optimal harvesting threshold with respect to changes in the volatility of the underlying interest rate dynamics is now illustrated in Figure 2 under the assumptions that $b=0.1, a=0.045 b, \gamma=0.75$, and $\mu=0.03$

Finally, the set of admissible volatility coefficients is illustrated in Figure 3 under the assumption that $b=0.1, a=0.045 b$, and $\mu=0.03$. Since the set of admissible volatility pairs is formed by the region below the curves, we observe that higher risk aversion decreases the set of admissible volatility pairs. 


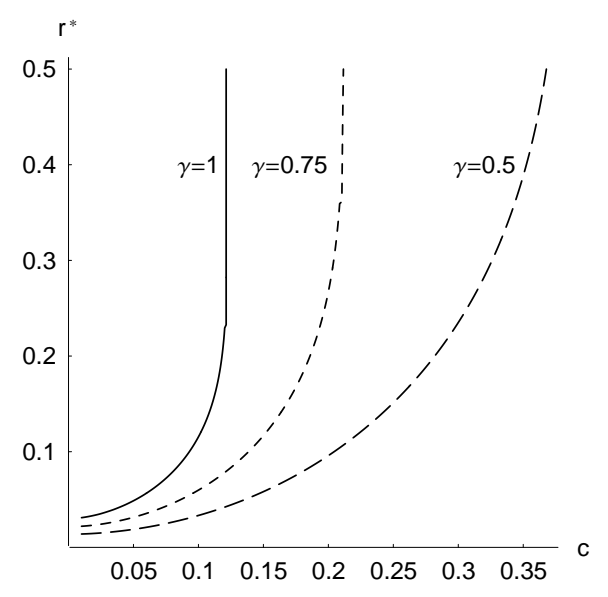

Figure 1: The optimal rotation threshold as a function of interest rate volatility $c$

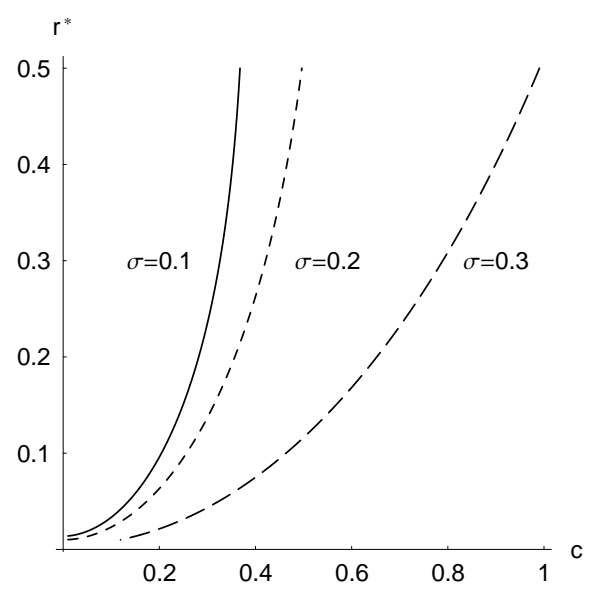

Figure 2: The optimal rotation threshold as a function of interest rate volatility $c$

\section{Conclusion}

In this paper we have used the Wicksellian single rotation framework to study the issue of forest rotation under variable and stochastic interest rate when forest stand value is also stochastic and landowners are risk averse. In order to accomplish this task, we have modelled the stochastic and intertemporally fluctuating interest rate as a parametrized mean-reverting process by applying the Cox-Ingersoll-Ross model of interest rate - which is well-known in financial economics and lies in conformity with empirics - and forest stand value as a geometric Brownian motion. We provided an explicit solution for the two-dimensional path-dependent optimal stopping problem and demonstrated that higher interest rate volatility increases 


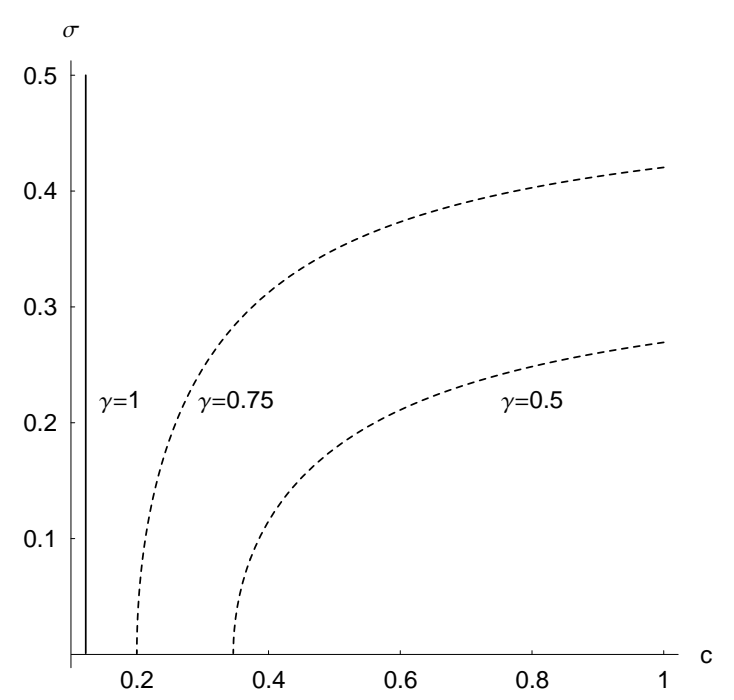

Figure 3: The set of admissible volatility pairs $(c, \sigma)$

the optimal harvesting threshold and thereby lengthens the expected rotation period, while higher relative risk aversion has the reverse effect. Interestingly, we also found that under risk aversion higher forest value volatility will have an accelerating effect on the optimal rotation policy by decreasing the harvesting threshold at which the harvesting opportunity should be optimally exercised. But under risk neutrality forest value volatility does not affect the optimal rotation policy. Finally, numerical illustrations indicate that the optimal harvesting threshold is a strictly convex function of the underlying interest rate process meaning that the expected length of the rotation period becomes higher at an increasing rate as the interest rate volatility increases. But under risk aversion higher forest value volatility will decrease the sensitivity of the optimal harvesting threshold with respect to the interest rate volatility.

Whether our conclusions remain valid in the Faustmannian ongoing rotation framework is an open question beyond the scope of this paper. Given the close connection of impulse control problems and optimal stopping theory (see e.g. Alvarez 2004) we are tempted to conjecture that our conclusions can be generalized to the Faustmann framework as well. The verification of this conjecture is an open issue for future research.

Acknowledgements: The research of Luis H. R. Alvarez has been supported by the Foundation for the Promotion of the Actuarial Profession, Finnish Insurance Society, and the Yrjö Jahnsson Foundation. Erkki Koskela thanks the Bank of Finland for hospitality and the Research Unit of Economic Structures and Growth (RUESG) in the University of Helsinki funded jointly by the Academy of Finland, University of Helsinki, Bank of Finland, Nokia 
Group, and the Yrjö Jahnsson Foundation.

\section{References}

[1] Abramowitz, M. and Stegun, I. A., eds., Handbook of mathematical functions, 1968, Dover Publications, New York.

[2] Alvarez, L. H. R. Reward functionals, salvage values, and optimal stopping, 2001, Mathematical Methods of Operations Research, 54, 315-337.

[3] Alvarez, L. H. R. Stochastic forest stand value and timber harvesting, 2004, SIAM Journal on Control and Optimization, 42, 1972-1993.

[4] Alvarez, L. H. R. and Koskela, E. On forest rotation under interest rate variability, 2003, International Tax and Public Finance, 10, 489-503.

[5] Alvarez, L. H. R. and Koskela, E. Wicksellian theory of forest rotation under interest rate variability, 2004, Journal of Economic Dynamics and Control, in press.

[6] Borodin, A. and Salminen, P. Handbook on Brownian motion - Facts and formulae, 2nd edition, 2002, Birkhauser, Basel.

[7] Björk, T. Arbitrage Theory In Continuous Time, 1998, Oxford UP, Somerset.

[8] Chang, F-R. On the elasticities of harvesting rules, 2004, Journal of Economic Dynamics and Control, in press.

[9] Clarke, H. R. and Reed, W. J. The tree-cutting problem in a stochastic environment, 1989, Journal of Economic Dynamics and Control, 13, 569-595.

[10] Clarke, H.R. and Reed, W.J Harvest decisions and asset valuations for biological resources exhibiting size-dependent stochastic growth, 1990, International Economic Review, 31, 147-169.

[11] Cochrane, J.H. Asset Pricing, 2001, Princeton University Press.

[12] Cox, J. C., Ingersoll, J. E., and Ross, S. A. A theory of the term structure of interest rates, 1985, Econometrica, vol. 53, 385-407.

[13] Insley, M. A real options approach to the valuation of a forestry investment, 2002, Journal of Environmental Economics and Management, 44, 471-492. 
[14] Johansson, P.-O. and Löfgren, K-G. The Economics of Forestry \& Natural Resources, 1985, Basil Blackwell.

[15] Merton, R. Optimum consumption and portfolio rules in a continuous-time model, 1971, Journal of Economic Theory, 3, 373-413.

[16] Øksendal, B. Stochastic differential equations: An introduction with applications, (Sixth Edition) 2003, Springer, Berlin.

[17] Plantinga, A. J. The optimal timber rotation: an option value approach, 1998, Forest Science, 44, 192-202.

[18] Reed, W. J. The effects of the risk of fire on the optimal rotation of a forest, 1984, Journal of Environmental Economics and Management, 11, 180-190.

[19] Samuelson, P. A. Economics of forestry in an evolving society, 1976, Economic Inquiry, 14, 466-492.

[20] Willassen, Y. The stochastic rotation problem: A generalization of Faustmann's formula to stochastic forest growth, 1998, Journal of Economic Dynamics and Control, 22, 573596. 


\section{A Proof of Theorem 2.1}

Proof. Integrating the ordinary differential equation $d r_{t}=\left(a-b r_{t}\right) d t$ from 0 to $t$ yields $r_{t}-r=a t-b \int_{0}^{t} r_{s} d s$ implying that $e^{-\int_{0}^{t} r_{s} d s}=e^{\left(r_{t}-r\right) / b-a t / b}$. On the other hand, since $X_{t}^{\gamma}=x^{\gamma} e^{\mu \gamma t}$ we find that

$$
\hat{V}_{\gamma}(x, r)=\sup _{t \geq 0}\left[e^{-\int_{0}^{t} r_{s} d s} \frac{1}{\gamma} X_{t}^{\gamma}\right]=\frac{1}{\gamma} x^{\gamma} e^{-r / b} \sup _{t \geq 0}\left[e^{(\mu \gamma-a / b) t+r_{t} / b}\right] .
$$

Given this observation (A.1), consider now the mapping $g(t)=e^{(\mu \gamma-a / b) t+r_{t} / b}$. Standard differentiation then yields $g^{\prime}(t)=\left(\mu \gamma-r_{t}\right) g(t)$ implying that $g^{\prime}(t) \gtreqless 0$ as long as $r_{t} \lesseqgtr \mu \gamma$. Combining this finding with the result $r_{t}=a / b+e^{-b t}(r-a / b)$ and the assumption $\mu \gamma<a / b$ then finally shows that the rotation date

$$
t^{*}=\inf \left\{t \geq 0: r_{t} \geq \mu \gamma\right\}=\ln \left(\frac{a-b \min (\mu \gamma, r)}{a-b \mu \gamma}\right)^{1 / b}
$$

is optimal. Inserting this date in (A.1) then yields (2.4).

\section{B Proof of Lemma 2.2}

Proof. It is well-known that the solution of the stochastic differential equation (2.3) reads as $X_{t}=x \exp \left(\left(\mu-\sigma^{2} / 2\right) t+\sigma \hat{W}_{t}\right)$. Moreover, we find by applying Itô's theorem to the mapping $r \mapsto e^{z r}$ that

$$
e^{-\frac{1}{2}\left(z^{2} c^{2}-2 z b\right) \int_{0}^{t} r_{s} d s}=e^{z\left(r-r_{t}\right)+z a t} M_{t}
$$

where

$$
M_{t}=\exp \left(\int_{0}^{t} z c \sqrt{r_{s}} d W_{s}-\frac{1}{2} \int_{0}^{t} z^{2} c^{2} r_{s} d s\right)
$$

is a positive exponential martingale. Thus, choosing $z=A$ implies that the discount factor can be re-expressed as

$$
e^{-\int_{0}^{t} r_{s} d s}=e^{A\left(r-r_{t}\right)+A a t} M_{t} .
$$

Given this observation, we find that the present value of the utility from the forest stand value $X_{t}$ can be expressed as

$$
e^{-\int_{0}^{t} r_{s} d s} \frac{1}{\gamma} X_{t}^{\gamma}=\frac{1}{\gamma} x^{\gamma} e^{A\left(r-r_{t}\right)+A a t+\delta(\gamma) t} \hat{M}_{t} M_{t}
$$

where $\delta(\gamma)=\mu \gamma+\sigma^{2} \gamma(\gamma-1) / 2$, and $\hat{M}_{t}=e^{\sigma \gamma \hat{W}_{t}-\frac{1}{2} \gamma^{2} \sigma^{2} t}$ is a positive exponential martingale. Consequently, we find that the path-dependent optimal rotation problem (2.1) can be reexpressed as an ordinary path-independent optimal stopping problem

$$
V_{\gamma}(x, r)=\frac{1}{\gamma} x^{\gamma} e^{A r} \sup _{\tau} \mathbf{E}_{r}\left[e^{(\delta(\gamma)+a A) \tau-A r_{\tau}} \hat{M}_{\tau} M_{\tau}\right]
$$


Defining the equivalent measure $\mathbb{Q}$ through the likelihood-ratio $\frac{d \mathbb{Q}}{d \mathbb{P}}=\hat{M}_{t} M_{t}$ we can now re-express (B.1) as

$$
V_{\gamma}(x, r)=\frac{1}{\gamma} x^{\gamma} e^{A r} \sup _{\tau} \mathbf{E}_{r}^{\mathbb{Q}}\left[e^{(\delta(\gamma)+a A) \tau-A r_{\tau}}\right]
$$

where the interest rate process $r_{t}$ evolves according to the dynamics described by the following stochastic differential equation

$$
d r_{t}=\left(a-\left(b-A c^{2}\right) r_{t}\right) d t+c \sqrt{r_{t}} d \tilde{W}_{t}, \quad r_{0}=r
$$

where $\tilde{W}_{t}$ is a standard Brownian motion under the equivalent measure $\mathbb{Q}$. However, given the strong uniqueness of a solution for the stochastic differential equation above (cf. Øksendal, 2003, p. 68) we finally find that the rotation problem (2.1) can be rewritten in the pathindependent form (2.5) defined under the objective measure $\mathbb{P}$.

\section{Proof of Theorem 2.3}

Proof. Since

$$
L_{\gamma}(r)=\mathbf{E}_{r}\left[e^{(\delta(\gamma)+a A) \tau-A \hat{r}_{\tau}}\right]
$$

is an ordinary path-independent optimal stopping problem of a linear diffusion and, therefore, can be solved by relying on ordinary variational inequalities, the alleged result is a direct implication of Theorem 3 in Alvarez 2001. It is, therefore, sufficient to determine the increasing fundamental solution of the ordinary second-order differential equation

$$
\frac{1}{2} c^{2} r u^{\prime \prime}(r)+\left(a-\left(b-c^{2} A\right) r\right) u^{\prime}(r)+(\delta(\gamma)+a A) u(r)=0 .
$$

Making the transformation $u(r)=v(\theta r)$, where $\theta \in \mathbb{R}$ is an unknown constant, and defining the variable $y=\theta r$ yields that

$$
y v^{\prime \prime}(y)+\left(\frac{2 a}{c^{2}}-\frac{2\left(b-A c^{2}\right)}{c^{2} \theta} y\right) v^{\prime}(y)+\frac{2(\delta(\gamma)+a A)}{\theta c^{2}} v(y)=0 .
$$

Choosing $\theta=2\left(b-A c^{2}\right) / c^{2}$, then finally implies that the differential equation can equivalently be expressed as

$$
y v^{\prime \prime}(y)+\left(\frac{2 a}{c^{2}}-y\right) v^{\prime}(y)-\frac{2(\delta(\gamma)+a A)}{A c^{2}-b} v(y)=0
$$

which is Kummer's differential equation. 


\section{Proof of Theorem 2.4}

Proof. Since $\delta^{\prime}(\gamma)=\mu-\frac{1}{2} \sigma^{2}+\sigma^{2} \gamma$ we observe that the condition $\mu \geq \frac{1}{2} \sigma^{2}$ implies that $\delta(\gamma)$ is an increasing function of the parameter $\gamma$. Consequently, if $\hat{\gamma} \geq \gamma \geq 0$ then the superharmonicity of the value function $L_{\hat{\gamma}}(r)$ and the inequality $L_{\hat{\gamma}}(r) \geq e^{-A r}$ yield the following inequalities

$$
L_{\hat{\gamma}}(r) \geq \mathbf{E}_{r}\left[e^{(\delta(\hat{\gamma})+a A) \tau_{n}} L_{\hat{\gamma}}\left(r_{\tau_{n}}\right)\right] \geq \mathbf{E}_{r}\left[e^{(\delta(\gamma)+a A) \tau_{n}} L_{\hat{\gamma}}\left(r_{\tau_{n}}\right)\right] \geq \mathbf{E}_{r}\left[e^{(\delta(\gamma)+a A) \tau_{n}-A r_{\tau_{n}}}\right],
$$

where $\tau_{n}$ is a sequence of almost surely finite stopping times converging to an arbitrary stopping time $\tau$. Letting $n \rightarrow \infty$ and invoking Fatou's theorem implies

$$
L_{\hat{\gamma}}(r) \geq \lim _{n \rightarrow \infty} \mathbf{E}_{r}\left[e^{(\delta(\gamma)+a A) \tau_{n}-A r_{\tau_{n}}}\right] \geq \mathbf{E}_{r}\left[e^{(\delta(\gamma)+a A) \tau-A r_{\tau}}\right]
$$

Since this inequality is valid for an arbitrary stopping time, it has to be valid for the optimal as well proving that $L_{\hat{\gamma}}(r) \geq L_{\gamma}(r)$ for all $r \in \mathbb{R}_{+}$. Given this observation, denote the continuation region where harvesting is suboptimal as $C_{\gamma}=\left\{r \in \mathbb{R}_{+}: L_{\gamma}(r)>e^{-A r}\right\}$. If $r \in C_{\gamma}$ then the inequality $L_{\hat{\gamma}}(r) \geq L_{\gamma}(r)>e^{-A r}$ implies that $r \in C_{\hat{\gamma}}$ as well and, therefore, that $C_{\gamma} \subseteq C_{\hat{\gamma}}$ which completes the proof of our theorem.

\section{E Proof of Theorem 2.5}

Proof. Since $\partial \delta(\gamma) / \partial \sigma=\sigma \gamma(\gamma-1)<0$ for all $\gamma \in(0,1)$ we observe that increased timber value volatility decreases the factor $\delta(\gamma)+a A$ and, therefore, decreases the value of the harvesting opportunity. Proving the alleged negativity of the sign of the relationship between increased timber value volatility and the optimal harvesting threshold is analogous with the proof of Theorem 2.4. 